\title{
An Inpatient Observational Study: Characteristics And Outcomes Of Avoidant/Restrictive Food Intake Disorder (ARFID) In Children And Adolescents In Japan
}

This article was published in the following Dove Press journal:

Neuropsychiatric Disease and Treatment

\author{
Isaku Kurotori ${ }^{1}$ \\ Katsutoshi Shioda' \\ Takaaki Abe' \\ Rika Kato' \\ Shizukiyo Ishikawa ${ }^{2}$ \\ Shiro Suda' \\ 'Department of Psychiatry, ${ }^{2}$ Department \\ of Medical Education Center, Jichi Medical \\ University, Tochigi, Japan
}

Purpose: To determine the clinical characteristics and course of severe avoidant/restrictive food intake disorder (ARFID) in hospitalized children and adolescents and compare them with those of patients with restricting-type anorexia nervosa (R-AN).

Patients and methods: We conducted a retrospective chart review of inpatients diagnosed with ARFID or R-AN based on the Diagnostic and Statistical Manual of Mental Disorders, Fifth Edition, at Jichi Children's Medical Center Tochigi between April 1, 2007 and March 31, 2017. We compared the characteristics of the ARFID and R-AN patients at admission, during hospitalization, and after discharge.

Results: Both the ARFID ( $n=13)$ and R-AN ( $n=79)$ patients required hospitalization for their medically unstable state. The features of ARFID group included concern about the aversive consequences of eating and avoidance of eating due to sensory concerns. Significant differences were found at admission between ARFID and R-AN groups in age (10.7 vs 12.7 years), family history of mental disorders ( $46.2 \%$ vs $17.7 \%$ ), comorbid developmental disorders ( 6 vs 3 cases), and the time from onset to admission ( 3.9 vs 6.3 months). The body weight status, $\%$ ideal body weight (\%IBW), \% expected body weight (\%EBW), $<75 \%$ IBW rate, and $<75 \%$ EBW rate did not differ significantly between the two groups at admission or discharge. The duration of post-discharge outpatient follow-up treatment did not differ significantly between ARFID and R-AN groups (15.3 vs 18.4 months); however, ARFID group recovery rate was significantly higher than that of R-AN group ( $77 \%$ vs $43 \%$ ). The reasons that the patients with ARFID had significantly better outcomes than the R-AN patients remain unclear. Compared to those in previous studies, the present patients were younger and demonstrated better outcomes. Our results indicate that the body weight status is similar between ARFID and R-AN patients, but the ARFID patients achieved better outcomes.

Conclusion: These findings suggest that early onset in childhood, early disease recognition, and early intervention are important factors for achieving better outcomes for patients with ARFID.

Keywords: avoidant/restrictive food intake disorder, ARFID, restricting-type anorexia nervosa, children and adolescents, inpatient, recovery

\section{Introduction}

In the Diagnostic and Statistical Manual of Mental Disorders, Fifth Edition (DSM-5), avoidant/restrictive food disorder (ARFID) replaces "feeding disorder of infancy or early childhood" described in the DSM's fourth edition (DSM-IV-TR), ${ }^{2}$ which was a diagnosis restricted to children $\leq 6$ years old. In the DSM-5, the diagnosis of ARFID (Box 1) abolishes the age restriction, extending its clinical utility from childhood
Correspondence: Katsutoshi Shioda Department of Psychiatry, Jichi Medical University, 33 II-I Yakushiji, Shimotsuke, Tochigi 329-0498, Japan

Tel +8I-285-58-7364

Fax +8I-285-44-6I 98

Email kazs@jichi.ac.jp 
throughout the entire lifespan. ${ }^{3-7}$ According to the DSM-5, ARFID is characterized as being without fear of gaining weight or a disturbance in the perception of one's body shape, which is distinguishable from anorexia nervosa (AN). ARFID involves the avoidance of eating for other motives, such as concern over the sensory characteristics of food, fear of the consequences of eating (e.g., vomiting, choking, swallowing), or a lack of interest. Although ARFID is defined without an age restriction, it is commonly diagnosed in older children and young adolescents, but this does not mean that the diagnosis of ARFID is less common in adults compared to younger individuals. Adult patients with ARFID may not have been diagnosed properly and may not have received treatment due to a lack of traditional eating disorder symptoms and low weight loss. ${ }^{6,8}$

Two recent studies revealed that the food-related emotion of disgust is related to ARFID symptoms ${ }^{9,10}$ and that patients with ARFID experienced disgust associated with their reaction to food, such as fear of vomiting or choking; therefore, patients with ARFID restrict their food intake, leading to one or more of the following: weight loss/growth faltering, nutritional

Box I DSM-5 Diagnosis Of Avoidant/Restrictive Food Intake Disorder

\begin{tabular}{|c|}
\hline $\begin{array}{l}\text { A. An eating or feeding disturbance (e.g., an apparent lack of interest } \\
\text { in eating or food, avoidance based on the sensory characteristics of } \\
\text { food, or concern about aversive consequences of eating) as } \\
\text { manifested by persistent failure to meet appropriate nutritional and/ } \\
\text { or energy needs associated with one (or more) of the following: }\end{array}$ \\
\hline $\begin{array}{l}\text { I. Significant weight loss (or failure to achieve expected weight gain or } \\
\text { faltering growth in children). }\end{array}$ \\
\hline 2. Significant nutritional deficiency. \\
\hline 3. Dependence on enteral feeding or oral nutritional supplements. \\
\hline $\begin{array}{l}\text { 4. Marked interference with psychosocial functioning. } \\
\text { B. The disturbance is not better explained by lack of available food or } \\
\text { by associated culturally sanctioned practice. }\end{array}$ \\
\hline $\begin{array}{l}\text { C. The eating disturbance does not occur exclusively during the } \\
\text { course of anorexia nervosa or bulimia nervosa, and there is no } \\
\text { evidence of a disturbance in the way in which one's body weight or } \\
\text { shape is experienced. }\end{array}$ \\
\hline $\begin{array}{l}\text { D. The eating disturbance is not attributable to a concurrent medical } \\
\text { condition or not better explained by another mental disorder. When } \\
\text { the eating disturbance occurs in the context of another condition or } \\
\text { disorder, the severity of the eating disturbance exceeds that routinely } \\
\text { associated with the condition or disorder and warrants additional } \\
\text { clinical attention. }\end{array}$ \\
\hline
\end{tabular}

Notes: Reprinted with permission from the Diagnostic and Statistical Manual of Mental Disorders, Fifth Edition, (Copyright O2013). American Psychiatric Association. All Rights Reserved.' deficiencies, supplement dependence, and psychosocial impairment (Box 1). ${ }^{1}$ Thus, ARFID often obstructs the quality of daily activities, such as going to school or taking part in social activities.

In several studies published in $2014,{ }^{11-14}$ the charts of patients diagnosed based on the DSM-IV were retrospectively reviewed using the DSM-5. The studies reported a $5 \%-23 \%$ relative rate of ARFID among the patients recruited from a pediatric tertiary care program that treats adolescents diagnosed primarily with a food-avoidant emotional disorder, childhood anorexia nervosa, selective eating, or an eating disorder not otherwise specified. Research has also indicated that adolescents with ARFID are significantly younger, more often male, have a longer duration of hospitalization, and are more likely to require enteral nutrition compared to adolescents with AN. ${ }^{12,14,15}$ Although some study results differ, ${ }^{16}$ when compared with other eating disorders, patients with ARFID tend to have a longer duration of untreated illness before medical intervention, poorer responses to weight gain, and a higher rate of treatment discontinuation during follow-up. ${ }^{12,13}$ There are reports that the long-term outcome of ARFID is equivalent to that of $\mathrm{AN},{ }^{17,18}$ but another research group contended that the outcome of ARFID is better than that of $\mathrm{AN},{ }^{19}$ and thus there is no consensus.

Due to chronic nutritional insufficiency, patients with ARFID often become medically unstable, exhibiting electrolyte abnormality, bradycardia, arrhythmia, liver dysfunction, and hypoglycemia, which result in hospitalization. Strandjord et al reported that among inpatients with ARFID, those who required hospitalization because of nutritional insufficiency had remission rates similar to those of patients with AN. ${ }^{15}$ However, few studies have focused on medically unstable patients hospitalized with severe weight loss due to ARFID ${ }^{20}$ and the clinical outcomes of children and adolescents with ARFID have not been elucidated. The clinical symptoms of ARFID seem to be similar to those of restricting-type AN (R-AN). Therefore, to help improve our understanding of this devastating disorder, we compared ARFID and R-AN inpatients and investigated their hospitalization clinical courses.

\section{Patients And Methods}

\section{Participants}

In this retrospective chart review study, we examined all children and adolescents aged $\leq 16$ years old who were admitted to the child and adolescent psychiatric ward of Jichi Children's Medical Center Tochigi between April 1, 2007 and March 31, 2017. We identified 13 Japanese 
patients with ARFID ( 2 boys, 11 girls) and 79 with R-AN (1 boy, 78 girls) based on the DSM- $5^{1}$ criteria (Box 1) who required hospitalization for their medically unstable state. The children's issues included severe malnutrition, dehydration, electrolyte disturbance, cardiac dysrhythmia, physiological instability, disturbance in growth and development, failure of outpatient treatment, acute food refusal, acute medical complications of malnutrition, and acute psychiatric emergencies. ${ }^{21}$ The patients were assessed by trained psychiatrists, and their progress was charted weekly. Our psychiatric personnel diagnosed ARFID and other psychiatric disorders based on the DSM-5 criteria. Patients admitted before 2013 and initially diagnosed with "feeding disorder of infancy or early childhood" or "eating disorder not otherwise specified" based on the DSM-IV-TR were re-examined with the DSM-5, and it was confirmed that all of the 13 patients with ARFID met the criteria of ARFID. We examined the patients' clinical manifestations of ARFID, i.e., apparent lack of interest in eating, avoidance due to sensory concerns, and concern over aversive consequences of eating. ${ }^{1}$ The exclusion criteria were: binge eating/the purging type of $\mathrm{AN}$, a diagnosis of other eating disorders, hospitalization without a medically unstable state, and any cases with a questionable diagnosis. This study was performed in accordance with the ethical standards detailed in the Declaration of Helsinki and approved by the Jichi Medical University (JMU) Ethics Committee. Since this research was a retrospective chart review with no patient contact or intervention, the risk to our study subjects was deemed minimal, and a waiver of the requirement for patient or parents' consent was obtained from the JMU Ethics Committee. The patients' personal data were protected by anonymization, and opting out was also an option if a patient or parents did not want to participate in the research plan; this option was published on the homepage of JMU's Department of Psychiatry.

\section{Treatment Procedure}

On admission, both the ARFID and R-AN patients were initially treated with biological management and nutritional rehabilitation. In parallel with these treatments, supportive psychotherapy was performed to allay their fear of eating, swallowing, or vomiting and to guarantee their safety during their hospitalization. We undertook a multidisciplinary approach consisting of behavioral management, cognitive behavioral therapy, nutritional counseling, and meal supervision.
The medical care team consisted of psychiatrists, pediatricians, nursing staff, psychologists, and psychiatric social workers. Nasogastric feeding was used when a patient could not eat and was losing weight rapidly. We considered prescription medicine for the symptoms of comorbid developmental, anxiety, obsessive-compulsive, and/or depressive disorders (Table 1). The patients in the adolescent psychiatric ward had the opportunity to attend a hospital-based school and participate in daily creative and physiotherapy programs, including group therapy. Individual and family counseling, a psychoeducational program, and nutritional support were performed weekly.

Patients were considered in remission when their behavioral eating patterns were restored and they could maintain their target weight for $\geq 2$ weeks. After a patient achieved remission, he or she was discharged and started weekly outpatient treatment sessions administered by trained psychiatrists to maintain their target body weight (BW) and normalize their eating patterns. The outpatient treatment sessions were also focused on subsequent better adaptation at home and upon returning to school.

\section{Measures}

Patient charts were reviewed by trained psychiatrists (IK and TA), and eating disorder diagnoses were determined retrospectively using the DSM- $5^{1}$ criteria. We retrospectively examined the patients' clinical data via a chart review. The information obtained included age at admission, sex, BW, height, body mass index (BMI), family history of mental disorders, comorbidity of developmental disorders, time between onset and admission, number of hospital stays, BW increase during hospitalization, use of nasogastric feeding, rehospitalization rate, and full recovery rate. Full recovery was defined as a BMI $>3$ rd percentile (i.e., the 3rd percentile BMI based on gender and age, defined using the Japanese Society for Pediatric Endocrinology [JSPE] BMI-for-age growth table ${ }^{22}$ ) in addition to meeting the full remission criteria or none of the ARFID or R-AN criteria, according to the DSM-5, for $>6$ months, after which no further treatment was provided.

As a child's weight and height increase during growth and development, the interpretation of a child's BMI should be considered in relation to those of other children of the same sex and age. Therefore, the percent expected body weight (\%EBW) and the percentage of ideal body weight (\%IBW) are more suitable measures than the BMI to evaluate a child's BW status. Each patient's \%EBW ${ }^{15,23}$ at admission was calculated by dividing the patient's BMI 


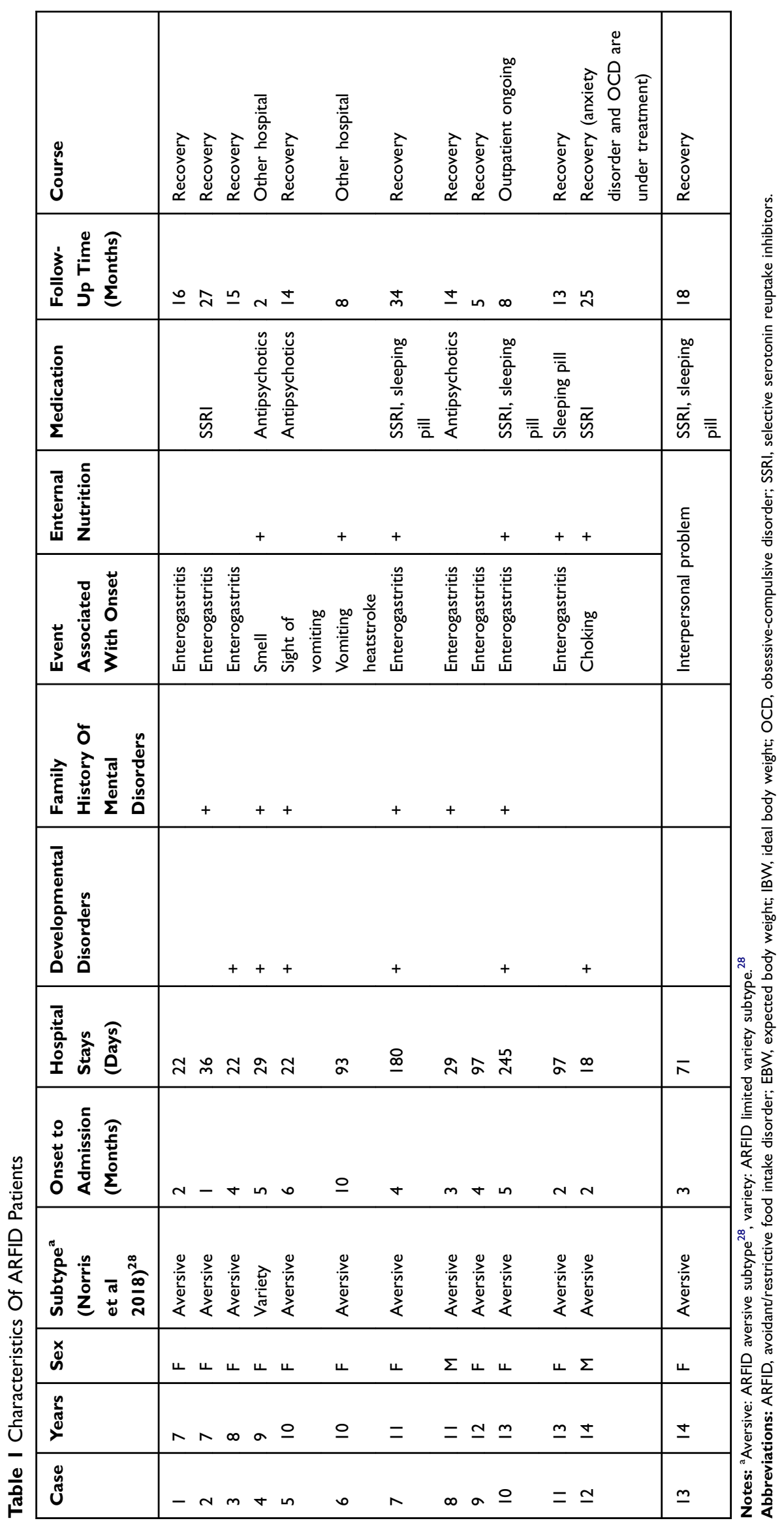


by the median BMI for his or her age and multiplying by $100(\% \mathrm{EBW}=$ patient $\mathrm{BMI} /$ median $\mathrm{BMI} \times 100)$. The median BMI, i.e., the 50th percentile BMI based on gender and age, was defined using the JSPE BMI-for-age growth table. $^{22}$ The $\% \mathrm{IBW}^{24}$ was calculated by dividing the patient's BW by the standard weight for the patient's sex, age, and height in Japanese children and multiplying that value by $100(\% \mathrm{IBW}=$ patient $\mathrm{BW} /$ standard $\mathrm{BW} \times 100)$. The standard weight was determined using the JSPE calculation formula. $^{25}$

Individuals with $<75 \%$ EBW or IBW have been reported to be physically at risk and thus should be hospitalized. ${ }^{24,26,27}$ Herein, we, therefore, provide the rate of patients with $<75 \% \mathrm{EBW}$ or IBW at admission and at discharge.

\section{Statistical Analysis}

We evaluated the mean (standard deviation, SD) age, BMI, $\% \mathrm{EBW}, \% \mathrm{IBW}$, time between onset and admission, number of hospital days, the percentages ( $\%$ ) of males, patients with $<75 \% \mathrm{EBW}$, and those with $<75 \%$ IBW, as well as the family history of mental disorders, development problems, enteral nutrition, rehospitalization, and recovery. We compared variables between the two groups (ARFID vs R-AN) using Welch's $t$-test for continuous variables and Fisher's exact test for categorical variables. P-values $<0.05$ were considered significant. All statistical analyses were performed using IBM SPSS Statistics for Windows, ver. 24.7 .

\section{Results}

We used the DSM-5 to re-examine the 13 patients diagnosed with ARFID based on the DSM-IV-TR, and all 13 patients were confirmed to have met the ARFID criteria. Of the patients with ARFID, 12 (92.3\%) presented with concern over the aversive consequences of eating as the most prominent clinical feature (aversive subtype ${ }^{28}$ ), and one patient $(7.7 \%)$ presented with sensory concerns as the most prominent clinical feature (variety subtype ${ }^{28}$ ). None of the ARFID patients showed an apparent lack of interest in eating (intake subtype ${ }^{28}$ ). The previous medical conditions or life events that the patients had experienced which might have been associated with the development of ARFID included enterogastritis $(n=8)$, vomiting due to heatstroke $(n=1)$, the sight of the patient's mother's vomiting $(n=1)$, interpersonal problems $(n=1)$, choking $(n=1)$, and the smell of a school lunch $(\mathrm{n}=1)$ (Table 1).

The mean age in ARFID group (10.7 years) was significantly younger than that in the R-AN group $(12.7$ years; $\mathrm{p}<0.05$, effect size 1.31). ARFID and R-AN groups did not differ significantly in the $\%$ EBW, $\%$ IBW, $<75 \%$ EBW, or $<75 \%$ IBW rate at admission or at discharge; they also did not differ in the change of $\% \mathrm{EBW}$ and $\%$ IBW during hospitalization. Although the BMI values at admission did not differ significantly between the two groups, the BMI values at discharge were significantly lower in ARFID (13.9 \pm 1.5$)$ group compared to the R-AN (15 \pm 1.4$)$ group $(\mathrm{p}<0.05$, effect size 0.84$)$ (Tables $2-5)$. A family history of mental disorders (46.2\% vs $17.7 \%$ ) and developmental disorders (6 vs 3 cases) was both significantly more common in ARFID group compared to R-AN group $(\mathrm{p}<0.05$, effect size 0.24 ; $\mathrm{p}<0.001$, effect size 0.5).

The mean length of time between onset and admission in ARFID group (15.3 months) was significantly shorter than that in R-AN group ( 18.4 months) $(\mathrm{p}<0.01$, effect size $0.6)$. The use of nasogastric feeding did not differ significantly between the two groups. Although no significant differences were seen in the duration of the follow-up period or in the rehospitalization rate, the rate of recovery was significantly higher in ARFID group (77\%) compared to the R-AN group $(43 \%)(\mathrm{p}<0.05$, effect size 0.24$)$. One patient in ARFID group who showed no ARFID symptoms had to continue receiving outpatient treatment for

Table 2 Patient Comparison Between ARFID And AN Groups (average)

\begin{tabular}{|c|c|c|c|c|}
\hline \multirow[t]{2}{*}{ Characteristic } & ARFID Group $(n=\mid 3)$ & AN Group $(n=79)$ & \multirow[t]{2}{*}{$P_{\text {-value }}{ }^{a}$} & \multirow[t]{2}{*}{ Effect Size ${ }^{b}$} \\
\hline & \multicolumn{2}{|l|}{ Average (SD) } & & \\
\hline Age at admission (years) & $10.7(2.5)$ & I2.7 (I.4) & $<0.05$ & 1.31 \\
\hline BMI at admission $\left(\mathrm{kg} / \mathrm{m}^{2}\right)$ & $13.0(1.5)$ & $13.5(1.5)$ & 0.24 & 0.37 \\
\hline \% EBW at admission (\%) & $74.4(8.5)$ & $71.6(7.1)$ & 0.28 & 0.39 \\
\hline \% IBW at admission (\%) & $74.3(9.0)$ & $70.0(6.7)$ & 0.12 & 0.61 \\
\hline
\end{tabular}

Notes: ${ }^{a}$ Welch's $t$ test, ${ }^{\mathrm{b}}$ Cohen's $d$.

Abbreviations: ARFID, avoidant/restrictive food intake disorder; AN, anorexia nervosa; BMI, body mass index; EBW, expected body weight; IBW, ideal body weight; SD, standard deviation. 
Table 3 Comparisons Between Patients In The ARFID And AN Groups

\begin{tabular}{|c|c|c|c|c|}
\hline \multirow[t]{2}{*}{ Characteristic } & ARFID Group $(n=\mid 3)$ & AN Group $(n=79)$ & \multirow[t]{2}{*}{ P-Value $^{a}$} & \multirow[t]{2}{*}{ Effect Size ${ }^{b}$} \\
\hline & \multicolumn{2}{|l|}{ Number (\%) } & & \\
\hline Sex (male) & $2(15.4)$ & $\mathrm{I}(\mathrm{I} .3)$ & 0.05 & 0.28 \\
\hline$<75 \%$ EBW at admission & $8(6 \mid .5)$ & $56(70.9)$ & 0.53 & 0.07 \\
\hline$<75 \%$ IBW at admission & $9(69.2)$ & $59(74.7)$ & 0.74 & 0.04 \\
\hline Family history of mental disorders & $6(46.2)$ & $14(17.7)$ & $<0.05$ & 0.24 \\
\hline Developmental problems & $6(46.2)$ & $3(3.8)$ & $<0.001$ & 0.5 \\
\hline
\end{tabular}

Notes: ${ }^{\text {a } F i s h e r ' s ~ e x a c t ~ t e s t, ~}{ }^{\mathrm{b}}$ Cramer's V.

Abbreviations: ARFID, avoidant/restrictive food intake disorder; AN, anorexia nervosa; BMI, body mass index; EBW, expected body weight; IBW, ideal body weight.

Table 4 Comparison Of Treatment Course Between ARFID And AN Groups

\begin{tabular}{|c|c|c|c|c|}
\hline \multirow[t]{2}{*}{ Characteristic } & ARFID Group $(n=\mid 3)$ & AN Group $(n=79)$ & \multirow[t]{2}{*}{ P-Value $^{\text {a }}$} & \multirow[t]{2}{*}{ Effect Size ${ }^{b}$} \\
\hline & \multicolumn{2}{|l|}{ Average (SD) } & & \\
\hline Time from onset to admission (months) & $3.9(2.3)$ & $6.3(4.1)$ & $<0.01$ & 0.60 \\
\hline Hospital stays (days) & $73.9(69.9)$ & $85.7(56.3)$ & 0.57 & 0.20 \\
\hline Follow-up time (month) & $15.3(9.1)$ & $18.4(15.0)$ & 0.32 & 0.22 \\
\hline BMI at discharge $\left(\mathrm{kg} / \mathrm{m}^{2}\right)$ & $13.9(1.5)$ & $15.0(1.4)$ & $<0.05$ & 0.84 \\
\hline \% EBW increase during hospitalization (\%) & $4.9(4.5)$ & $7.9(6.7)$ & 0.06 & 0.45 \\
\hline \% IBW increase during hospitalization (\%) & $4.9(4.6)$ & $7.7(6.5)$ & 0.08 & 0.44 \\
\hline \% EBW at discharge (\%) & $79.3(6.5)$ & $79.5(6.7)$ & 0.96 & 0.01 \\
\hline \% IBW at discharge (\%) & $79.2(6.9)$ & $77.7(6.2)$ & 0.48 & 0.24 \\
\hline
\end{tabular}

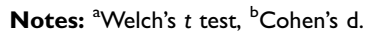

Abbreviations: ARFID, avoidant/restrictive food intake disorder; AN, anorexia nervosa; BMI, body mass index; EBW, expected body weight; IBW, ideal body weight; SD, standard deviation.

anxiety disorder for $>1$ year. Another two patients in ARFID group discontinued their outpatient care prematurely. One patient with the features of avoidance due to sensory concerns was discharged with a nasogastric tube and transferred to another hospital because the patient's family lived a long distance from our hospital. Another patient had an address change and moved to another hospital, without remission. None of the patients were reclassified or underwent the transition from ARFID to AN during the follow-up period.

\section{Discussion}

Our retrospective analyses of 13 adolescent patients with ARFID and 79 adolescent patients with R-AN revealed that age, a family history of mental disorder, the presence of one or more developmental disorders, the length of time between onset and admission, the BMI at discharge, and the recovery rate were significantly different between the two patient groups. The factors that did not differ significantly between the groups were the \%EBW, \%IBW, $<75 \%$ EBW rate, and $<75 \%$ IBW rate at admission and at

Table 5 Comparison Of Treatment Course Between The ARFID And AN Groups

\begin{tabular}{|l|l|l|l|l|}
\hline \multirow{2}{*}{ Characteristic } & \multicolumn{2}{|l|}{ ARFID Group (n=13) } & \multirow{2}{*}{ AN Group (n = 79) $^{*}$} & \multirow{2}{*}{ Effect Size $^{\mathbf{b}}$} \\
\cline { 2 - 4 } & \multicolumn{2}{|l|}{ Number (\%) } & & \\
\hline Enteral nutrition & $6(46.2)$ & $29(36.7)$ & 0.55 & 0.07 \\
$<75 \%$ EBW at discharge & $5(38.5)$ & $20(25.3)$ & 0.33 & 0.10 \\
$<75 \%$ IBM at discharge & $5(38.5)$ & $26(32.9)$ & 0.53 & 0.04 \\
Rehospitalization & I (7.7) & $24(30.4)$ & 0.08 & 0.18 \\
Recovery & $10(77.0)$ & $34(43.0)$ & $<0.05$ & 0.24 \\
\hline
\end{tabular}

Notes: ${ }^{\text {a }}$ isher's exact test, ${ }^{\text {b } C r a m e r ' s ~ V . ~}$

Abbreviations: ARFID, avoidant/restrictive food intake disorder; AN, anorexia nervosa; BMI, body mass index; EBW, expected body weight; IBW, ideal body weight. 
discharge; the BMI at admission, the duration of the follow-up period, and the changes of $\% \mathrm{EBW}$ and $\% \mathrm{IBW}$ during hospitalization.

Twelve of our ARFID patients were classified as having the aversive subtype of ARFID, ${ }^{28}$ and one patient was classified as having variety subtype. ${ }^{28}$ Norris et al found that patients with aversive-type ARFID, i.e., those with concern over the aversive consequences of eating, were more likely to be hospitalized for tertiary care. ${ }^{28}$ It was reported that patients with aversive-type ARFID often experience fear (e.g., fear of choking, pain, or nausea), ${ }^{28}$ and Zickgraf et $\mathrm{al}^{29}$ reported that the presentation of fear was associated with a more severe and acute onset of ARFID. We thus speculate that most of the ARFID patients in the present study had aversive-type ARFID.

Regarding our findings that age, family history of mental disorders, and presence of developmental disorders in ARFID group differed significantly from those in R-AN group, Nicely et al reported ${ }^{14}$ that patients with ARFID had more developmental disorders than patients with $\mathrm{AN}$, and our findings are consistent with that report. A family history of mental disorders was more common in ARFID group compared to R-AN group; this result is not consistent with those of previous studies. ${ }^{11,15}$ Our patients with ARFID were significantly younger than the R-AN patients, which is consistent with findings of previous studies, but our groups' gender ratio, duration of hospitalization, and the need for enteral nutrition are not in agreement with earlier investigations. ${ }^{12,13,15,29}$

Our comparison of the patient groups' treatment courses revealed that the patients with ARFID showed significantly lower BMI values at discharge plus the absence of between-group significant differences in BW status, including the $\% \mathrm{IBW}$ and $\% \mathrm{EBW}$ at admission and discharge, the increase in \%IBW and \%EBW during hospitalization, and the rates of $<75 \%$ IBW and $<75 \%$ EBW at admission and discharge. Considering these results, there were no significant differences between the groups in the patients' weight status at admission or discharge or in the improvement of their weight status during admission. These results demonstrating similar discharge weight statuses in ARFID patient and R-AN patient are consistent with those of previous studies. ${ }^{17,28,29}$

Compared to the patients in similar studies, ${ }^{18,29,30}$ our ARFID patients exhibited a poorer weight status, younger age, and a shorter time to hospitalization. Nevertheless, 10 (77.0\%) of the 13 patients in ARFID group achieved a full recovery after discharge. This rate is significantly higher than that of R-AN group $(43 \% ; \mathrm{p}<0.05)$. Some research groups have reported no difference between the improvement of

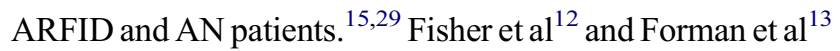
reported that patients with ARFID demonstrated a longer duration of untreated illness before medication, a poor weight-gain response, and a higher rate of treatment discontinuation. These two research groups reported that their patients with ARFID exhibited poor improvement compared to their AN patients, and they noted that ARFID is difficult to diagnose early (patients with ARFID usually have less weight loss than AN patients). ${ }^{12,13}$

In contrast, Nakai et al described the outcomes of ARFID in adolescents and adults (15-40 years old) in Japan; the rate of full recovery (though their definition of full recovery differed from ours) among patients with ARFID was $51.9 \%$, compared with $35.5 \%$ for patients with AN. ${ }^{19}$ Our present study's follow-up duration was shorter and our patients were younger compared to those in the study by Nakai et al, ${ }^{19}$ which also reported a shorter duration of illness in their younger patients with ARFID compared to those with AN. ${ }^{19}$ The early detection of ARIFID is thus important.

The reasons why the patients in ARFID group had significantly superior outcomes compared to R-AN group remain unclear. One potential reason may be that weight loss in ARFID is unintentional, and, as is the case with any phobic avoidance, the patients usually want to resume their normal activities. There is no ego-syntonic component to food restriction in ARFID, especially fear-based ARFID, and there is no body-image distortion. Our findings suggest that onset in early childhood, early recognition of the disorder, and early intervention may be important factors for achieving better outcomes in ARFID.

This study has several limitations. It was a retrospective analysis of a small sample size that was imbalanced between the two diagnostic groups $(n=13, n=79)$. There were no standardized assessments or precise psychological variables in our study. However, to our knowledge, no other studies have been conducted on the treatment course and outcomes of hospitalized children and adolescents with exceptionally low weight due to ARFID. Prospective, randomized control trials and multicenter studies are required to reveal the more complete picture of ARFID and to develop treatments, as there is no established treatment for ARFID.

In addition, although ARFID might have an entirely different pathology than other eating disorders, diagnostic difficulties and overlaps remain throughout the adolescent 
growth period. A reclassification or transition from ARFID to AN may eventually be required. To more clearly understand the variations in ARFID, a long-term prospective cohort study is needed.

\section{Conclusions}

The results of our present analyses demonstrated the clinical characteristics and course of hospitalized children and adolescents with ARFID. The patients in this study had a poorer weight status and a younger average age compared to the patients in previous studies. Our findings suggest that there is no significant difference in the body weight status between ARFID and R-AN patients, but our ARFID group achieved significantly better outcomes than our R-AN group. The reasons for this remain to be determined. Onset during early childhood, early recognition of the disorder, early intervention, and adequate intensive care may lead to better outcomes for patients with ARFID.

\section{Abbreviations}

AN, anorexia nervosa; ARFID, avoidant/restrictive food intake disorder; BMI, body mass index; BW, body weight; DSM-5, The Diagnostic and Statistical Manual of Mental Disorders; EBW, expected body weight, Fifth Edition; IBW, ideal body weight; R-AN, restricting type anorexia nervosa.

\section{Acknowledgments}

We thank the Clinical Research Support Team Jichi (iCRST) and the staff of the JMU Support Center for Clinical Investigation (J-SENSATION).

\section{Disclosure}

The authors report no conflicts of interest in this work.

\section{References}

1. American Psychiatric Association. Diagnostic and Statistical Manual of Mental Disorders (DSM- $\left.{ }^{\circledR}\right)$, Fifth Edition. Arlington (VA): American Psychiatric Association; 2013. ISBN: 978-0-89042-554-1.

2. American Psychiatric Association. Diagnostic and Statistical Manual of Mental Disorders. 4th. text rev. Washington (DC): American Psychiatric Association; 2000. ISBN-13: 978-0890420621.

3. Bryant-Waugh R. Avoidant restrictive food intake disorder: an illustrative case example. Int $J$ Eat Disord. 2013;46(5):420-423. doi:10.1002/eat.22093

4. Katzman DK, Stevens K, Norris M. Redefining feeding and eating disorders: what is avoidant/restrictive food intake disorder? Paediatr Int Child Health. 2014;19(8):445-446. doi:10.1093/pch/19.8.445

5. Norris ML, Spettigue WJ, Katzman DK. Update on eating disorders: current perspectives on avoidant/restrictive food intake disorder in children and youth. Neuropsychiatr Dis Treat. 2016;19:213-218. doi:10.2147/NDT.S82538
6. Becker KR, Keshishian AC, Liebman RE, et al. Impact of expanded diagnostic criteria for avoidant/restrictive food intake disorder on clinical comparisons with anorexia nervosa. Int $J$ Eat Disord. 2019;52:230-238. doi:10.1002/eat.22988

7. Lieberman M, Houser ME, Voyer AP, et al. Children with avoidant/ restrictive food intake disorder and anorexia nervosa in a tertiary care pediatric eating disorder program: A comparative study. Int $J$ Eat Disord. 2019;52:239-245. doi:10.1002/eat.23027

8. Zickgraf HF, Franklin ME, Rozin P. Adult picky eaters with symptoms of avoidant/restrictive food intake disorder: comparable distress and comorbidity but different eating behaviors compared to those with disordered eating symptoms. $J$ Eat Disord. 2016;4:26. doi:10.1186/s40337-016-0110-6

9. Menzel JE, Reilly EE, Luo TJ, et al. Conceptualizing the role of disgust in avoidant/restrictive food intake disorder: implications for the etiology and treatment of selective eating. Int J Eat Disord. 2019;52:462-465. doi:10.1002/eat.23006

10. Zickgraf HF, Murray HB, Kratz HE, et al. Characteristics of outpatients diagnosed with the selective/neophobic presentation of avoidant/restrictive food intake disorder. Int J Eat Disord. 2019;52:367377. doi:10.1002/eat.23013

11. Norris ML, Robinson A, Obeid N, et al. Exploring avoidant/restrictive food intake disorder in eating disordered patients: A descriptive study. Int J Eat Disord. 2014;47(5):495-499. doi:10.1002/eat.22217

12. Fisher MM, Rosen DS, Ornstein RM, et al. Characteristics of avoidant/restrictive food intake disorder in children and adolescents: a "new disorder" in DSM-5. J Adolesc Health. 2014;55(1):49-52. doi:10.1016/j.jadohealth.2013.11.013

13. Forman SF, McKenzie N, Hehn R, et al. Predictors of outcome at 1 year in adolescents with DSM-5 restrictive eating disorders: report of the national eating disorders quality improvement collaborative. J Adolesc Health. 2014;55(6):750-756. doi:10.1016/j. jadohealth.2014.06.014

14. Nicely TA, Lane-Loney S, Masciulli E, et al. Prevalence and characteristics of avoidant/restrictive food intake disorder in a cohort of young patients in day treatment for eating disorders. $J$ Eat Disord. 2014;2:21-30. doi:10.1186/s40337-014-0021-3

15. Strandjord SE, Sieke EH, Richmond M, et al. Avoidant/restrictive food intake disorder: illness and hospital course in patients hospitalized for nutritional insufficiency. J Adolesc Health. 2015;57(2):673678. doi:10.1016/j.jadohealth.2015.08.003

16. Ornstein RM, Essayli JH, Nicely TA, et al. Treatment of avoidant/ restrictive food intake disorder in a cohort of young patients in a partial hospitalization program for eating disorders. Int J Eat Disord. 2017;50:1067-1074. doi:10.1002/eat.22737

17. Lange CRA, Ekedahl Fjertorp H, Holmer R, et al. Long-term followup study of low-weight avoidant restrictive food intake disorder compared with childhood-onset anorexia nervosa: psychiatric and occupational outcome in 56 patients. Int $J$ Eat Disord. 2019;52:435-438. doi:10.1002/eat.23038

18. Bryson AE, Scipioni AM, Essayli JH, et al. Outcomes of low-weight patients with avoidant/restrictive food intake disorder and anorexia nervosa at long-term follow-up after treatment in a partial hospitalization program for eating disorders. Int $J$ Eat Disord. 2018;51 (5):470-474. doi:10.1002/eat.22853

19. Nakai Y, Nin Y, Noma S, et al. Clinical presentation and outcome of avoidant/restrictive food intake disorder in a Japanese sample. Eat Behav. 2017;24:49-53. doi:10.1016/j.eatbeh.2016.12.004

20. Thomas JJ, Lawson EA, Micali N, et al. Avoidant/restrictive food intake disorder: A three-dimensional model of neurobiology with implications for etiology and treatment. Curr Psychiatry Rep. 2017;19(8):54. doi:10.1007/s11920-017-0795-5

21. Golden NH, Katzman DK, Kreipe RE, et al. Eating disorders in adolescents: position paper of the society for adolescent medicine. $J$ Adolesc Health. 2003;33(6):496-503. doi:10.1016/s1054-139x(03) 00326-4 
22. Japanese Society for Pediatric Endocrinology. Available from: http:// jspe.umin.jp/medical/files/fuhyo5.pdf (in Japanese). Accessed October 10, 2019

23. Peebles R, Lesser A, Park CC, et al. Outcomes of an inpatient medical nutritional rehabilitation protocol in children and adolescents with eating disorders. J Eat Disord. 2017;5:7. doi:10.1186/s40337017-0134-6

24. American Academy of Pediatrics Committee on Adolescence. Identifying and treating eating disorders. Pediatrics. 2003;111 (1):204-211. doi:10.1542/peds.111.1.204.

25. Japanese Society for Pediatric Endocrinology. Available from: http:// jspe.umin.jp/medical/taikaku.html (in Japanese). Accessed October 10, 2019.

26. Schwartz BI, Mansbach JM, Marion JG, et al. Variations in admission practices for adolescents with anorexia nervosa: a North American sample. $J$ Adolesc Health. 2008;43(5):425-431. doi:10.1016/j. jadohealth.2008.04.010
27. Le Grange D, Doyle PM, Swanson SA, et al. Calculation of expected body weight in adolescents with eating disorders. Pediatrics. 2012;129(2):e438-46. doi:10.1542/peds.2011-1676

28. Norris ML, Spettigue W, Hammond NG, et al. Building evidence for the use of descriptive subtypes in youth with avoidant restrictive food intake disorder. Int J Eat Disord. 2018;51(2):170-173. doi:10.1002/ eat. 22814

29. Zickgraf HF, Lane-Loney S, Essayli JH, et al. Further support for diagnostically meaningful ARFID symptom presentations in an adolescent medicine partial hospitalization program. Int $J$ Eat Disord. 2019;52:402-409. doi:10.1002/eat.23016

30. Makhzoumi SH, Schreyer CC, Hansen JL, et al. Hospital course of underweight youth with ARFID treated with a meal-based behavioral protocol in an inpatient-partial hospitalization program for eating disorders. Int J Eat Disord. 2019;52(4):428-434. doi:10.1002/ eat.23049

\section{Publish your work in this journal}

Neuropsychiatric Disease and Treatment is an international, peerreviewed journal of clinical therapeutics and pharmacology focusing on concise rapid reporting of clinical or pre-clinical studies on a range of neuropsychiatric and neurological disorders. This journal is indexed on PubMed Central, the 'PsycINFO' database and CAS, and is the official journal of The International Neuropsychiatric Association (INA). The manuscript management system is completely online and includes a very quick and fair peer-review system, which is all easy to use. Visit http://www.dovepress.com/testimonials.php to read real quotes from published authors. 